\title{
Selective Molecular Recognition of Polycyclic Aromatic Hydrocarbons (PAHs) on Calix[4]arene-Functionalized Ag Nanoparticles by Surface-Enhanced Raman Scattering
}

\author{
P. Leyton, ${ }^{\dagger}$ S. Sanchez-Cortes, ${ }^{*}$, J. V. Garcia-Ramos, ${ }^{\ddagger}$ C. Domingo, ${ }^{\ddagger}$ M. Campos-Vallette, ${ }^{\dagger}$ \\ C. Saitz, ${ }^{\S}$ and R. E. Clavijo ${ }^{\dagger}$ \\ University of Chile, Faculty of Sciences, P.O. Box 653, Santiago, Chile, Instituto de Estructura de la Materia, \\ CSIC, Serrano 121, Madrid-28006, Spain, and Department of Organic and Physical Chemistry, Faculty of \\ Chemical and Pharmaceutical Sciences, University of Chile, Olivos 1007, P.O. Box 233, Santiago, Chile
}

Received: May 13, 2004; In Final Form: September 3, 2004

\begin{abstract}
Trace concentrations of polycyclic aromatic hydrocarbons (PAHs) have been successfully detected using surface-enhanced Raman scattering (SERS) spectroscopy. For such purpose, new SERS substrates have been developed, consisting of Ag nanoparticles, either in suspension or immobilized on glass, obtained by a new method and covered by adsorbed self-assembled calix[4]arene molecules. Among the assayed calix[4]arenes, the 25,27-dicarboethoxy-26,28-dihydroxy-p-tert-butylcalix[4]arene host molecule displays analytical selectivity to the PAH guest systems bearing four benzene rings, mainly pyrene. The host-guest interaction mechanism seems to take place through a $\pi-\pi$ stacking interaction leading to a charge transfer between the complex and the metallic surface, which may also induce a notable influence on the surface charge of the metallic nanoparticle.
\end{abstract}

\section{Introduction}

Molecular recognition of bowl-shaped hosts with a welldefined inner cavity capable of size-selective molecular encapsulation is a topic of current interest in supramolecular chemistry with interesting applications in sensors design. ${ }^{1}$ Among these host molecules, calixarenes have recently attracted much attention. $^{2}$

Calixarenes are a class of synthetic cyclooligomers formed via a phenol-formaldehyde condensation. They exist in a "cuplike" shape with a defined upper and lower rim and a central annulus (Figure 1). Calixarenes have interesting applications as host molecules as a result of their preformed cavities. Moreover, they display different conformations such as cones, partial cones, 1,2-alternates, and 1,3-alternates, ${ }^{2-4}$ which may condition the calixarene applications as host molecules. By changing the chemical groups of the upper and/or lower rim, it is possible to prepare different derivatives with differing selectivities for various guest ions and molecules. The adsorption and self-assembled monolayer formation of calixarenes is a prerequisite for the application of calixarenes in sensor devices. However, the adsorption and organization of calixarene molecules on substrates have been scarcely studied on metal surfaces, where charge-transfer phenomena can take place with interest for optical sensing. ${ }^{5-8}$

Polycyclic aromatic hydrocarbons (PAHs, Figure 2) are a group of different chemical compounds with a condensed multibenzene structure. PAHs are important environmental pollutants formed during the incomplete burning of coal, oil, and gas or other organic substances such as tobacco or charbroiled meat. ${ }^{9}$ PAHs can be found as a mixture of different related molecular compounds in air, soil, and water due to both natural processes and human activity. Because many of them

* To whom correspondence should be addressed. E-mail: imts158@ iem.cfmac.csic.es. Fax: + 349156455 57. Phone: + 34915616800 .

Faculty of Sciences, University of Chile.

$\doteqdot$ Instituto de Estructura de la Materia.

$\S$ Department of Organic and Physical Chemistry, University of Chile. a)

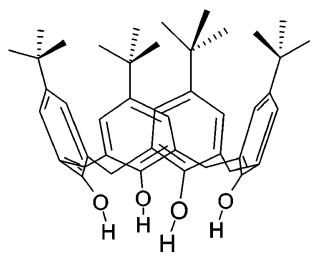

b)

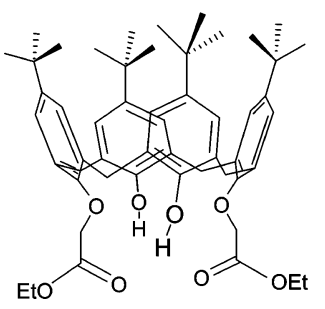

c)

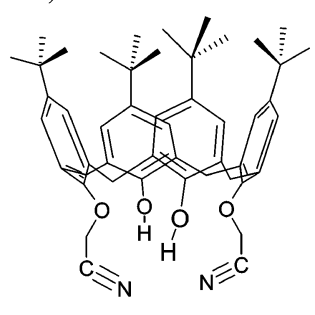

Figure 1. Molecular structure of calix[4]arenes: (a) TOHC, (b) DCEC, and (c) DCNC.

have been reported to be strong carcinogens, ${ }^{10-13}$ it is important to find an effective and selective method to detect them.

The chemical analysis of PAHs has been centered so far on chromatographic methods. ${ }^{14}$ In a number of recent works, Raman spectroscopy has been also applied in the detection of PAHs by using the SERS (surface-enhanced Raman spectroscopy) technique. ${ }^{15,16}$ In latter works, metal electrodes or metal island films were employed as substrates. However, the direct detection of PAHs by these methods is rather nonselective. In a recent work, we have published the surface-enhanced vibrational spectra (SERS and surface-enhanced infrared) of nitroPAH derivatives 1-nitropyrene and 2-nitrofluorene, ${ }^{17}$ in which we demonstrated the existence of an effective interaction of the adsorbate with the metal through the nitro group.

SERS is now a useful analytical technique that has been extensively employed in the identification and most probable orientation of molecules adsorbed onto a surface. The high 
a)<smiles>c1cc2ccc3cccc4ccc(c1)c2c34</smiles>
b)

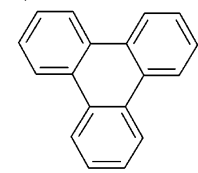

d)<smiles>c1ccc2cc3ccccc3cc2c1</smiles>

e)<smiles>c1cc2ccc3ccc4ccc5ccc6ccc1c1c2c3c4c5c61</smiles>

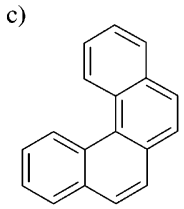

f)

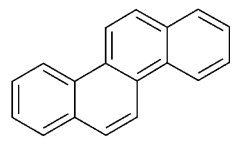<smiles>c1ccc2c(c1)ccc1cc3c(ccc4ccccc43)cc12</smiles>

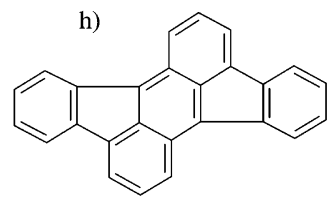

Figure 2. Molecular structure of (a) pyrene (PYR), (b) triphenylene (TP), (c) benzo[c]phenantrene (BcP), (d) anthracene (ANT), (e) coronene (COR), (f) chrysene (CHR), (g) dibenzoanthracene (DBA), and $(\mathrm{h})$ rubicene (RUB).

sensitivity of this tecnique is mainly due to the giant electromagnetic enhancement on nanostructured metal surfaces, ${ }^{18}$ although a part of the total enhancement is also attributed to a resonance effect associated with adsorbate-metal charge transfer. ${ }^{19}$

Few works have been devoted so far to the combined use of calixarenes and SERS in the application of these hosts to molecular recognition. ${ }^{5,20}$ The studies carried out up to now on the adsorption and self-assembly of calixarenes on metal surfaces mainly deal with thiol-functionalized calixarenes., ${ }^{5,20-23}$ However, other calixarene derivatives could also be effectively employed as hosts in the selective molecular recognition of PAHs using the SERS technique. In this work, we report the application of several calix[4]arenes with different chemical functional groups in the lower rim for the selective recognition of large PAHs molecules (Figure 2): anthracene (ANT), dibenzoanthracene (DBA), pyrene (PYR), benzo[c]phenantrene $(\mathrm{BcP})$, triphenylene (TP), coronene (COR), chrysene (CHR), and rubicene (RUB).

This is the first time that calixarenes have been used in the detection of PAHs by using vibrational spectroscopy and that nonthiolic calixarenes have been employed to functionalize a metal surface in the design of a film for optical sensing. The use of nanoparticles of $\mathrm{Ag}$ in suspension or immobilized on a substrate in the detection of large PAHs with environmental and toxicological relevance is also reported for the first time.

\section{Experimental Section}

Synthesis of Calix[4]arenes. The calixarenes employed for this study were (Figure 1) 25,26,27,28-tetrahydroxy-p-tert-butylcalix[4]arene (TOHC); 25,27-dicarboethoxy-26,28-dihydroxyp-tert-butylcalix[4]arene (DCEC), and 25,27-dicyano-26,28dihydroxy-p-tert-butylcalix[4]arene (DCNC). These calixarenes were synthesized by us following published procedures ${ }^{23}$ and are functionalized with four tert-butyl groups in the upper rim, whereas the two last calixarenes have a different functionalization in the lower rim with an alternate 1,3 substitution.

Preparation of the Metal Surfaces and Samples for SERS. Anthracene (ANT), dibenzoanthracene (DBA), pyrene (PYR), benzo $[c]$ phenantrene $(\mathrm{BcP})$, triphenylene $(\mathrm{TP})$, coronene $(\mathrm{COR})$, chrysene (CHR), and rubicene (RUB) were purchased from

\section{SCHEME 1}

\section{a) IMMOBILIZATION}

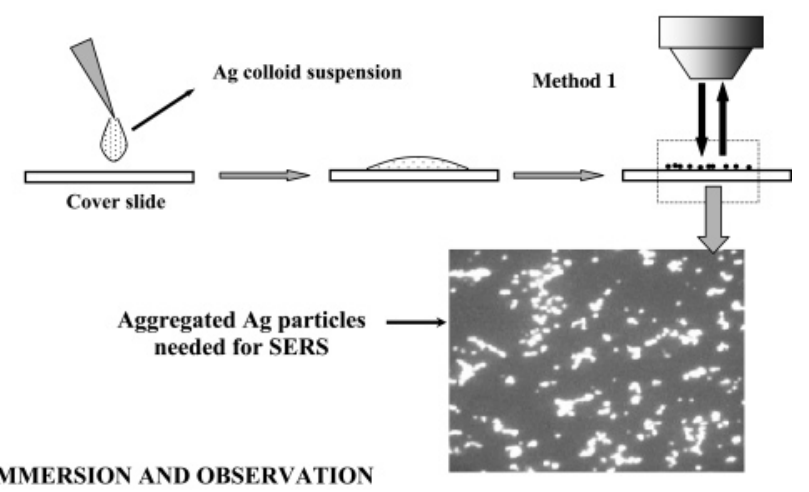

b) IMMERSION AND OBSERVATION

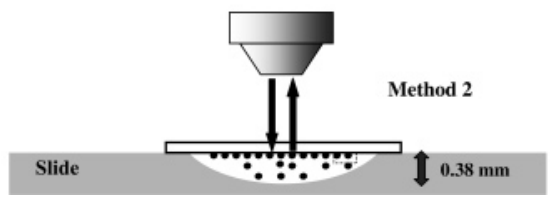

Aldrich and Merck and used as received. Solutions of these compounds in acetone (99\%) were prepared to a final concentration of $10^{-2} \mathrm{M}$.

Colloidal silver nanoparticles were prepared by using hydroxylamine hydrochloride as a reducing agent. ${ }^{24}$ These nanoparticles have the advantage, with respect to the citrate $\mathrm{Ag}$ colloid, of a more uniform distribution of size and shape together with the absence of excess citrate and its oxidation products, which could interfere with the SERS measurements. ${ }^{25}$ In addition, this colloid has better electrochemical properties on the surface for the detection of PAHs because the total negative charges, mainly due to the residual chloride ions, should be much lower than in the case of citrate and borohydride colloids. In the latter cases, anions bearing more than one negative charge are adsorbed on the surface, increasing the overall nanoparticle negative charge. However, these chloride-covered nanoparticles present improved adherence properties. This fact facilitates their immobilization on glass, giving rise to films provided with a uniform distribution of metal particles tightly linked to the glass by a simple deposition of an aliquot of the colloidal solution on a glass slide.

SERS measurements on Ag colloid suspensions were performed by adding aliquots of the calixarene in acetone to 500 $\mu \mathrm{L}$ of the silver colloid up to the desired concentration. In the case of the calixarene/PAHs complexes, aliquots of the PAHs solution, also in acetone, were then added to reach the final desired concentrations.

Metal films of the Ag nanoparticles were prepared for microSERS measurements by immobilizing the colloidal nanoparticles by two different methods. In both cases, the colloids were previously activated by the addition of $0.5 \mathrm{M}$ aqueous potassium nitrate up to a final concentration of $4 \times 10^{-2} \mathrm{M}$. This activation is needed to increase the nanoparticles SERS activity by properly modifying the particles' morphology. ${ }^{26}$ The first method consisted of the immobilization of the Ag nanoparticles on a glass cover slide by drying $20 \mu \mathrm{L}$ of the activated colloidal suspension at room temperature (Scheme 1a). Then, an aliquot of the calixarene in acetone was added to the immobilized film, and afterwards an aliquot of the PAHs solution also in acetone was also added. This sample was analyzed after drying the film. The second method consisted of the immobilization of the Ag nanoparticles already having the host-guest complex, prepared 


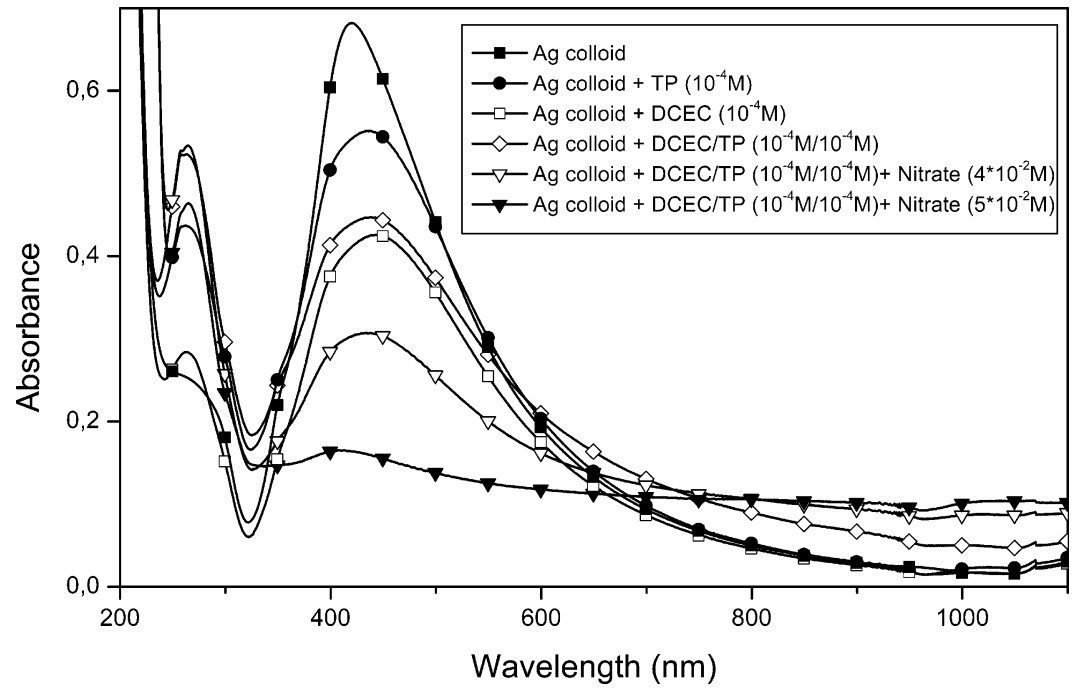

Figure 3. UV-vis extinction spectra of the Ag colloid employed to prepare the SERS samples and of the colloidal suspensions after the addition of the corresponding adsorbate indicated in the inset. All of the samples were diluted to $10 \%$.

following the method described above, by direct deposition on a cover glass (Scheme $1 b$ ). In this way, a large amount of analyte migrates to the surface, leading to a high surface coverage. Then, an aliquot of the original nonactivated colloid was placed on a glass slide provided with a shallow groove $(2-\mathrm{cm}$ diameter and 0.38-mm depth), and then the cover glass slide containing the dried Ag nanoparticles was placed on the groove with the side containing the dried colloid facing downward with respect to the suspension placed in the groove, as depicted in Scheme 1b. The second method provided more reproducible SERS spectra, and thus it was followed to obtain the SERS spectra shown in this work.

In the case of micro-SERS, the sensitivity is much higher than in macro experiments because of the smaller surface area studied by micro-Raman. Assuming that the applied aliquot $\left(10^{-5} \mathrm{M}\right.$ in PAHs) reached the whole surface of about $10^{8} \mu \mathrm{m}^{2}$ and that the analyzed surface is about $2 \mu \mathrm{m}^{2}$ when using a $100 \times$ objective in the micro-Raman, we have calculated that the total analyzed PAHs is approximately $10^{-3} \mathrm{pg}$. The analyte was uniformly spread on the metal surface as deduced from the similar Raman intensity registered in different points.

Instrumentation. FT-Raman spectra were obtained with a Bruker RFS 100/S instrument by using the line at $1064 \mathrm{~nm}$, provided by a Nd:YAG laser and a Ge detector cooled by liquid nitrogen. The resolution was set to $4 \mathrm{~cm}^{-1}$, and $180^{\circ}$ geometry was employed. The output laser power was $50 \mathrm{~mW}$. The solid samples were placed in a brass holder. Up to 1000 scans were accumulated.

The SERS spectra at $785 \mathrm{~nm}$ were measured with a Renishaw Raman Microscope System RM2000 equipped with a diode laser, a Leica microscope, and an electrically refrigerated CCD camera. The spectra shown here were obtained by using a $100 \times$ objective. The laser power in the sample was $2.0 \mathrm{~mW}$. The spectral resolution was set at $2 \mathrm{~cm}^{-1}$.

We noted that the choice of the excitation wavelength is important depending on the SERS experiment. In general, the best results were obtained for excitation lines in the red-nearinfrared regions. For the micro-SERS experiments, we used the 785-nm line (dispersive instrument) because at $1064 \mathrm{~nm}$ no SERS spectra could be obtained, most probably because of the different light absorption by the metal plasmons at the latter wavelength. The 1064-nm line (FT-Raman) leads to a selective enhancement of the Raman PAH signal, whereas in the visible $(785 \mathrm{~nm})$, the calixarene spectrum is more enhanced. We have also tried the 514-nm line, but in this case the sample is seriously damaged by the heat, mainly when using method 1 described above.

Samples for UV-visible absorption spectroscopy were prepared in the same way as those for the corresponding SERS spectra and were recorded in a Cintra 5 double-beam spectrophotometer. The colloidal suspensions were diluted to $10 \%$ in water and placed in 1-cm optical cuvettes.

\section{Results and Discussion}

Aggregation of Ag Nanoparticles: UV-Visible Extinction Spectra. Figure 3 shows the UV-visible extinction spectra of the hydroxylamine Ag colloid in the absence of any adsorbate and in the presence of the DCEC, TP, and DCEC/TP complexes, either with or without nitrate. For other PAHs molecules, the results were similar. As can be seen, both molecules separately induce a change in the extinction spectrum consisting mainly of the intensity decrease of the 420-nm band of the original colloid, which is slightly moved toward higher wavelengths (436 $\mathrm{nm})$. However, we did not observe a corresponding increase in the extinction in the near-infrared (NIR) region (i.e., above 800 $\mathrm{nm})$. In contrast, the DCEC/TP complex also induces a larger intensity decrease of the lower-wavelength band as well as a clear intensity increase of the extinction in the NIR region. This increase is due to the formation of large Ag aggregates ${ }^{26}$ that have a plasmon resonance in the NIR region and are very active from the SERS point of view because in them the electromagnetic field is greatly enhanced. Thus, the use of an excitation wavelength in this region should lead to a high SERS intensity, as will be shown below. This is the reason to employ the 785and 1064-nm excitation lines. This aggregation effect clearly indicates that the complex strongly modifies the electrical properties of the metal surface because of charge transfer induced by the formation of host-guest complexes adsorbed on the Ag nanoparticles as depicted in Scheme 2. Similar behavior is observed in the cases of PYR and BcP, but TP induces a larger aggregation than the other PAHs. Moreover, the subsequent addition of nitrate leads to further aggregation of the colloid with an increase in the extinction in the NIR region (Figure 3), although this aggregation does not have a significant influence on the SERS intensity.

Raman and SERS of Calix[4]arene Derivatives. We have centered our attention on DCEC calixarene because it renders 


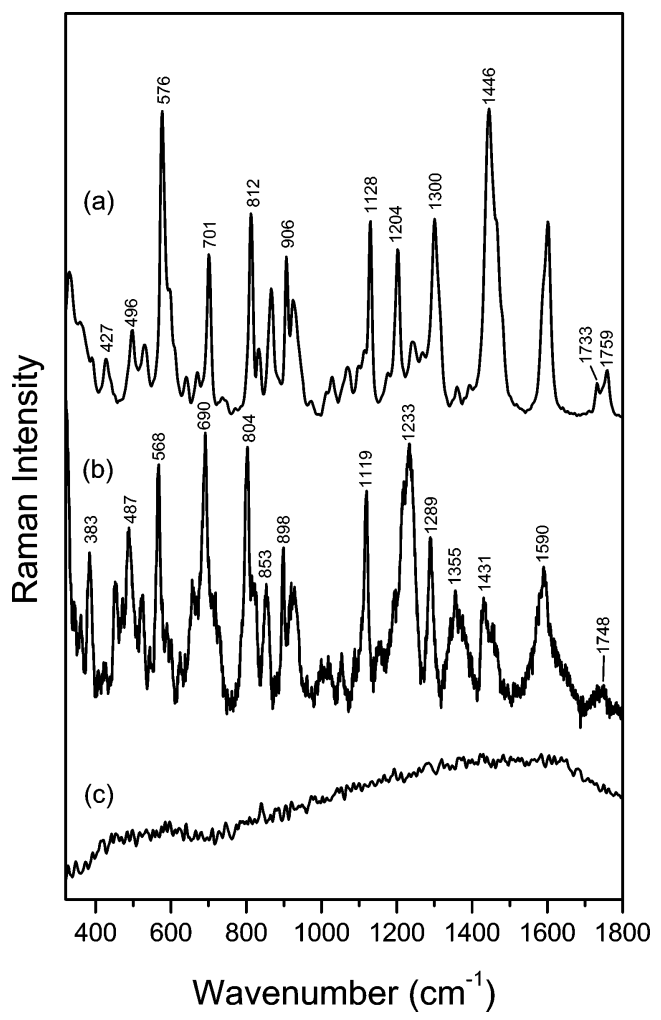

Figure 4. Raman spectrum of DCEC in the solid state exciting at (a) $1064 \mathrm{~nm}$ and SERS spectra of DCEC $\left(10^{-4} \mathrm{M}\right)$ on a Ag colloid at (b) 785 and (c) $1064 \mathrm{~nm}$.

\section{SCHEME 2: (A) Host/Guest Complex Formation on the Surface and (B) Aggregation Induced by the Complex Formation on the Surface}

A) Host/guest complex formation on the surface:

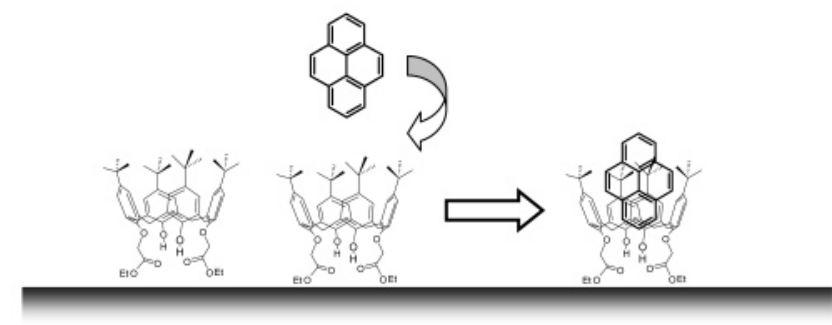

B) Aggregation induced by the complex formation on the surface:

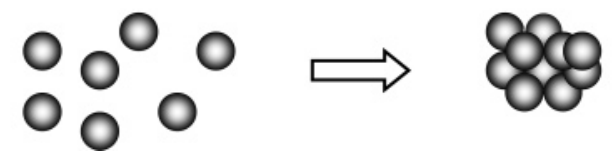

the highest SERS intensity in the series of calixarenes studied. Figure 4 shows the SERS spectra of DCEC in the solid state (Figure 4a) and adsorbed on Ag colloids (at $1064 \mathrm{~nm}$ ) and Ag colloidal films (at $785 \mathrm{~nm}$ ). The SERS intensity of all of the calixarenes in general is very weak when exciting at $1064 \mathrm{~nm}$ (Figure 4c), but the SERS intensity markedly increases at 785 $\mathrm{nm}$ (Figure $4 \mathrm{~b}$ ). The assignment of calixarene bands was made on the basis of already published works on the Raman spectra of similar calixarene compounds. ${ }^{27-29}$ The main features observed in the SERS of DCEC in relation to the solid spectrum are (a) the downward shift of the main ester $v(\mathrm{C}=\mathrm{O})$ stretching band from 1759 to $1748 \mathrm{~cm}^{-1}$ (Figure 4b), which is typical of metal complexes with esters and clearly suggests the existence of a chemical adsorbate/substrate interaction; ${ }^{30}$ (b) the intensification of two bands at 1355 and $1233 \mathrm{~cm}^{-1}$, which are assigned to $v(\mathrm{C}-\mathrm{O})$ vibrations coupled to ring-stretching vibrations and thus also related to the ester group; and (c) the weakening of the band centered at $1446 \mathrm{~cm}^{-1}$, attributed to $\mathrm{CH}_{3}$ and $\mathrm{CH}_{2}$ deformation vibrations of $t$-butyl groups on the upper rim, which also undergoes a marked shift downward. In general, the shifts and intensity enhancement observed for the bands attributed to the ester groups as well as the weakening and shift of tertbutyl bands indicate that the molecule interacts with the metal through the ester group, leading to a specific orientation where the tert-butyl groups are placed further from the surface.

The influence of the Ag surface on the ester group is evident and similar to that found for the binding of ester-functionalized calix[4]arenes with certain cations such as sodium. ${ }^{31}$ The adsorption of DCEC on the metal is also accompanied by a conformational change, as deduced from the differences in the 400-500- and $650-850-\mathrm{cm}^{-1}$ regions observed in the SERS spectrum in relation to the same regions in the solid. ${ }^{27-29}$ Thus, the metal induces a preferential conformation of DCEC when adsorbed on the metal surface.

The possible adsorption through the oxygen atoms of the ester groups is supported by the new band observed at $383 \mathrm{~cm}^{-1}$ in the SERS of DCEC, which we attribute to an $\mathrm{Ag}-\mathrm{O}$ vibration.

Raman and SERS of PAHs. The SERS of PAHs cannot be obtained on Ag colloids or in general on other metallic surfaces because these molecules do not manifest any affinity to be adsorbed on the metal surface. Our experience indicates that when depositing an aliquot of a PAH solution on metal-island films the molecule is not actually adsorbed once the solvent is evaporated because the PAH molecules remain as microcrystals. One of the advantages of micro-Raman is that the studied areas can be conveniently chosen. In this way, we have observed that the Raman spectrum of deposited microcrystals corresponds to the solid PAH, whereas no Raman spectrum could be obtained from the areas where there are no crystals (results not shown here).

This fact must be considered when obtaining macro-Raman or SERS from PAH solutions deposited and dried onto metal surfaces such as island films ${ }^{16}$ or electrodes. ${ }^{15}$ This means that it is necessary to functionalize the metal surface in order to attach the PAH molecules to the high enhancement points placed onto the metal and in order to ensure a suitable organization of the molecule on the surface.

Raman and SERS of Calix[4]arene/PAHs Complexes. The SERS of calixarene/PAHs complexes were obtained at two different excitation wavelengths: 1064 and $785 \mathrm{~nm}$ (Figures 5 and 6 , respectively). These two lines were selected to study the different components of the host/guest complex separately. As seen above, the calixarene bands are very weak at $1064 \mathrm{~nm}$, and the SERS spectrum is dominated by the spectrum of PAHs. Figure 5 displays the SERS spectra obtained for the PAHs rendering the most intense SERS signals (TP, PYR, and BcP) on DCEC, the host calixarene molecule leading to the most intense SERS spectra of the PAH molecules when exciting at $1064 \mathrm{~nm}$. At this wavelength, the minimum PAH concentration needed to obtain a SERS spectrum was $10^{-6} \mathrm{M}$.

The SERS spectra of PAHs (at $10^{-4} \mathrm{M}$ ) in the complex show important differences in relation to the corresponding Raman spectrum of the solid. In the case of $\mathrm{BcP}$ (Figure 5f) and the other PAHs, few things can be said about the FT-SERS of its complex with DCEC owing to the weakness of the SERS spectra obtained. However, significant differences between the SERS 


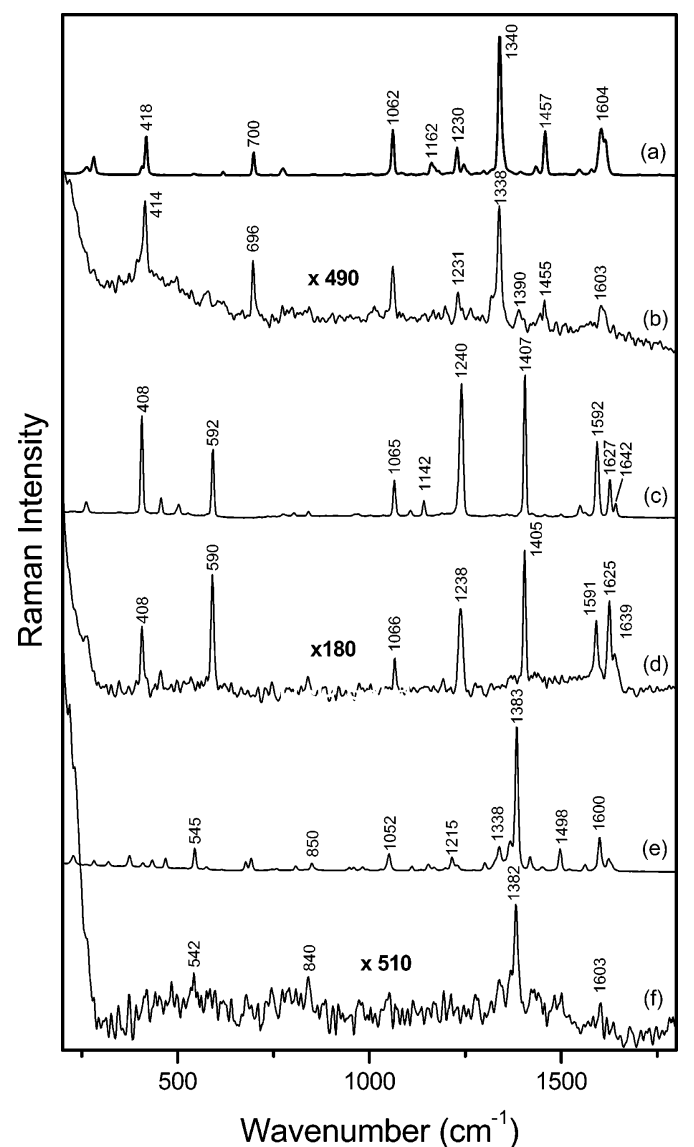

Figure 5. Raman spectra of $\mathrm{TP}, \mathrm{PYR}$, and $\mathrm{BcP}$ in the solid state (a, $\mathrm{c}$, and e, respectively) and SERS spectra of TP, PYR, and BcP $\left(10^{-4}\right.$ M) complexes with DCEC $\left(10^{-4} \mathrm{M}\right)$ on a Ag colloid (b, d, and f, respectively) at $1064 \mathrm{~nm}$.

spectrum of the complex and the corresponding Raman spectrum of the solid were observed for TP and PYR.

The SERS of TP (Figure 5b) shows weaker bands above 1300 $\mathrm{cm}^{-1}$ (at 1603, 1455, and $1338 \mathrm{~cm}^{-1}$ ) and a general and slight shift of the main bands downward. In addition, a relative intensity increase of the bands at 1390, 1315, 696, and $414 \mathrm{~cm}^{-1}$ and the weakening of the band at $1162 \mathrm{~cm}^{-1}$ are observed.

PYR undergoes even stronger changes in the complex with DCEC. There are several bands, those at 1639, 1625, 1405, and $590 \mathrm{~cm}^{-1}$, that undergo a higher enhancement in the SERS spectrum of the complex (Figure 5d). As in the case of TP, a general shift downward of the main bands and the weakening of the band at $1142 \mathrm{~cm}^{-1}$ (which may correspond to the band in TP at $1162 \mathrm{~cm}^{-1}$ ) can be observed. In general, all of the intensified bands correspond to symmetric $\mathrm{a}_{\mathrm{g}}$ modes, ${ }^{32-34}$ which are enhanced through a Franck-Condon resonance mechanism ${ }^{35}$ most probably associated with charge transfer in the complexsurface system, which also occurs for naphthalene interacting with $\mathrm{TOHC}^{36}$ or in complexes with other carbonaceous molecules (i.e., the fullerenes). ${ }^{37,38}$ In contrast, the band at 1591 $\mathrm{cm}^{-1}$, assigned to a nontotally symmetric $\mathrm{b}_{3 \mathrm{~g}}$ mode $\mathrm{e}^{32-34}$ undergoes a drastic weakening as expected by its geometry under resonance conditions. The charge transfer could be associated to the aggregation induced by the complex once adsorbed on the Ag nanoparticles (Scheme 2). On the basis of the above results, we have deduced a host-guest interaction mechanism through $\pi-\pi$ stacking between the aromatic systems of DCEC and PAHs.

Figure 6 shows the micro-SERS spectra of the DCEC/PYR complex at two different PYR concentrations: $10^{-4}$ (Figure 6b)

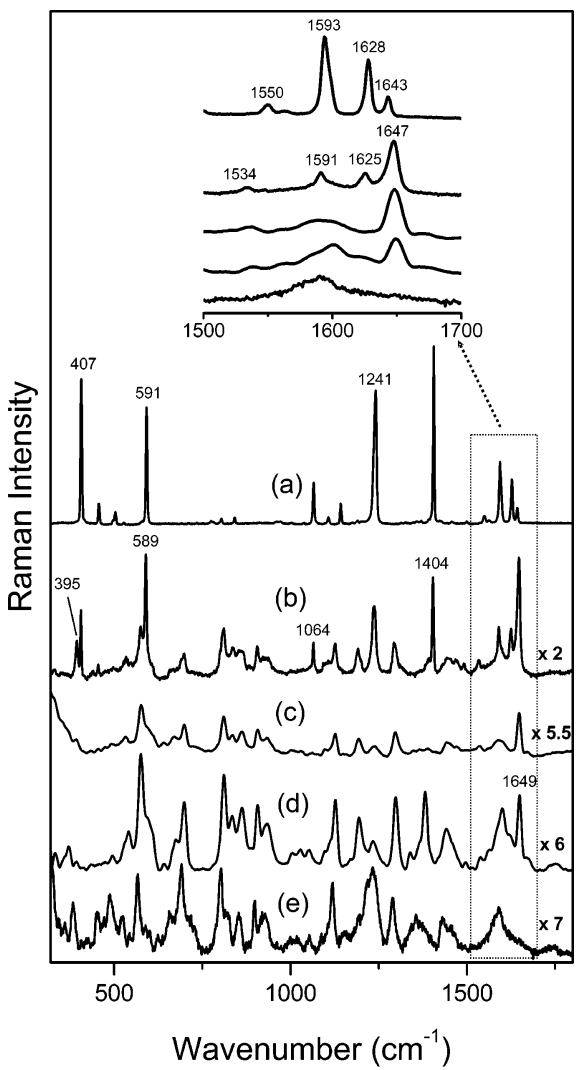

Figure 6. Micro-Raman spectra of PYR in (a) the solid state and microSERS spectra of (b) PYR $\left(10^{-4} \mathrm{M}\right)$, (c) PYR $\left(10^{-8} \mathrm{M}\right)$, (d) BcP $\left(10^{-6}\right.$ $\mathrm{M})$, and (e) DCEC $\left(10^{-4} \mathrm{M}\right)$. All of the spectra were obtained by exciting at $785 \mathrm{~nm}$.

and $10^{-8} \mathrm{M}$ (Figure $6 \mathrm{c}$ ) compared to the normal Raman of the solid PYR (Figure 6a). As can be seen, the SERS of PYR undergoes a remarkable change in the complex with DCEC. The complexation affects mainly the bands appearing in the $1650-1530-\mathrm{cm}^{-1}$ region, where a marked enhancement of the band at $1647 \mathrm{~cm}^{-1}$ is seen, together with a strong intensity decrease of the band at $1593 \mathrm{~cm}^{-1}$ and a relative increase of the small band at $1550 \mathrm{~cm}^{-1}$, which shifts to $1534 \mathrm{~cm}^{-1}$ in the complex. In the inset of Figure 6, the changes induced by the complexation are shown in more detail in comparison to the Raman of the solid PYR and DCEC. Also, another consequence of the complex formation is a general shift of the bands assigned to the ring-stretching vibrations toward lower frequencies except for the band appearing at $1647 \mathrm{~cm}^{-1}$, which could follow different behavior because it is a combination of bands at $1240\left(\mathrm{a}_{\mathrm{g}}\right)$ and $408\left(\mathrm{a}_{\mathrm{g}}\right) .^{33}$

The strong enhancement of the $1647-\mathrm{cm}^{-1}$ band is probably due to a resonance effect associated with the charge-transfer process occurring between the DCEC/PYR complex and the Ag nanoparticles. This assumption is corroborated by the fact that the resonance Raman spectrum of PYR obtained when excited in the UV ${ }^{39,40}$ also shows intense bands between 1625 and $1656 \mathrm{~cm}^{-1}$. The intensification of the band at $1647 \mathrm{~cm}^{-1}$ is more effective at lower relative concentrations of PYR in relation to that of the DCEC host. In fact, this band is still seen at a very low concentration $\left(10^{-12} \mathrm{M}\right)$ together with that at 1530 $\mathrm{cm}^{-1}$, suggesting a change in the complex structure or stoichiometry depending on the relative concentrations of ligand and host. The SERS of BcP also shows an intense band at 1649 $\mathrm{cm}^{-1}$ (Figure 6d), but in the case of TP (spectrum not shown), this band is not observed either in the SERS or in the resonance Raman spectrum. ${ }^{39}$ 


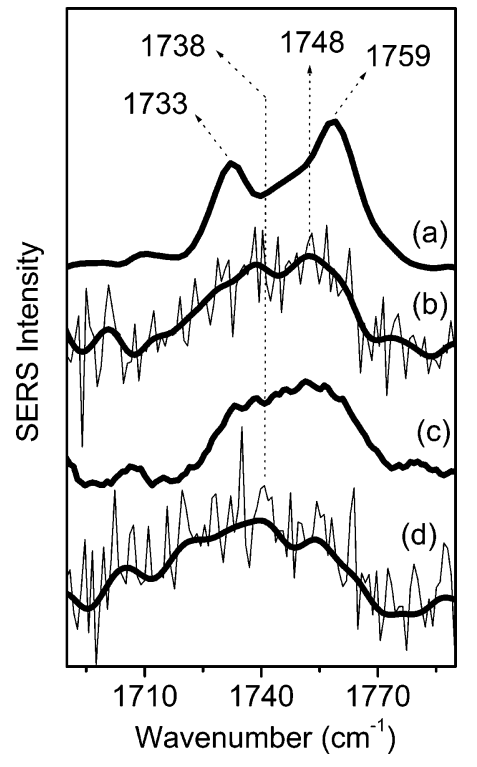

Figure 7. Detail of (a) the micro-Raman spectrum of DCEC showing the $v(\mathrm{C}=\mathrm{O})$ region in the solid state and (b) the micro-SERS spectra of DCEC $\left(10^{-4} \mathrm{M}\right)$ on a Ag colloid. SERS spectra of (c) BcP $\left(10^{-6}\right.$ $\mathrm{M})$ and of (d) PYR $\left(10^{-4} \mathrm{M}\right)$ complexes with DCEC $\left(10^{-4} \mathrm{M}\right)$. All of the spectra were obtained by exciting at $785 \mathrm{~nm}$.

However, the structure of DCEC in the complex seems to change markedly in the presence of PAHs as deduced from the SERS spectra of the complex with BcP (Figure 6e) or PYR (Figure $6 \mathrm{~b}$ and $\mathrm{c}$ ). In general, the SERS spectrum of DCEC in the complex is more similar to the Raman spectrum of solid, considering both the spectral pattern and the relative band intensities. This is probably due to a conformational change of the host upon complexation with the ligand, leading to a solidlike structure.

A more detailed analysis of the SERS spectra (Figure 7) reveals important changes in the ester $\mathrm{C}=\mathrm{O}$ stretching bands. The $\mathrm{C}=\mathrm{O}$ band splits in the solid spectrum of DCEC (Figures 7a), with two bands appearing at 1759 and $1733 \mathrm{~cm}^{-1}$. However, the $\mathrm{C}=\mathrm{O}$ stretching band appears centered at $1748 \mathrm{~cm}^{-1}$ in the SERS spectrum of DCEC (Figure $7 \mathrm{~b}$ ) and the complex with $\mathrm{BcP}$ (Figure 7c), whereas is seen at $1738 \mathrm{~cm}^{-1}$ in the complex with PYR (Figure 7d). As mentioned above, the changes observed in this band are related to the interaction of the calixarene with the Ag surface as also occurs in the interaction of DCEC with other metals ${ }^{29}$ and is typical of the complexes of esters with metals. ${ }^{30}$ Moreover, the larger shift downward of the ester $v(\mathrm{C}=\mathrm{O})$ bands as well as its weaker intensity in the case of the DCEC/PYR complex could be related to a stronger interaction of PYR in relation to $\mathrm{BcP}$. However, important changes are also observed in the bands around $1200 \mathrm{~cm}^{-1}$, also associated with the ester groups ( $\mathrm{C}-\mathrm{O}$ stretching modes), and in the bands below $1000 \mathrm{~cm}^{-1}$. Furthermore, the $\delta\left(\mathrm{CH}_{3}\right)$ and $\delta\left(\mathrm{CH}_{2}\right)$ bands, appearing in the $1450-1430-\mathrm{cm}^{-1}$ region, also undergo significant changes, confirming a conformational change in DCEC tert-butyl groups as a consequence of the interaction with the PAH.

Selectivity of the Calix[4]arenes/PAHs Interaction Detected by SERS. Figure $8 \mathrm{a}$ shows the normalized areas corresponding to the most intense SERS bands of PYR and BcP (those at 1405 and $1382 \mathrm{~cm}^{-1}$, respectively) in the complexes with the different calixarene molecules assayed in this study. As can be seen, PYR and BcP have different behavior as concerns the calixarene selectivity. PYR shows a much higher SERS intensity when it interacts with DCEC compared with
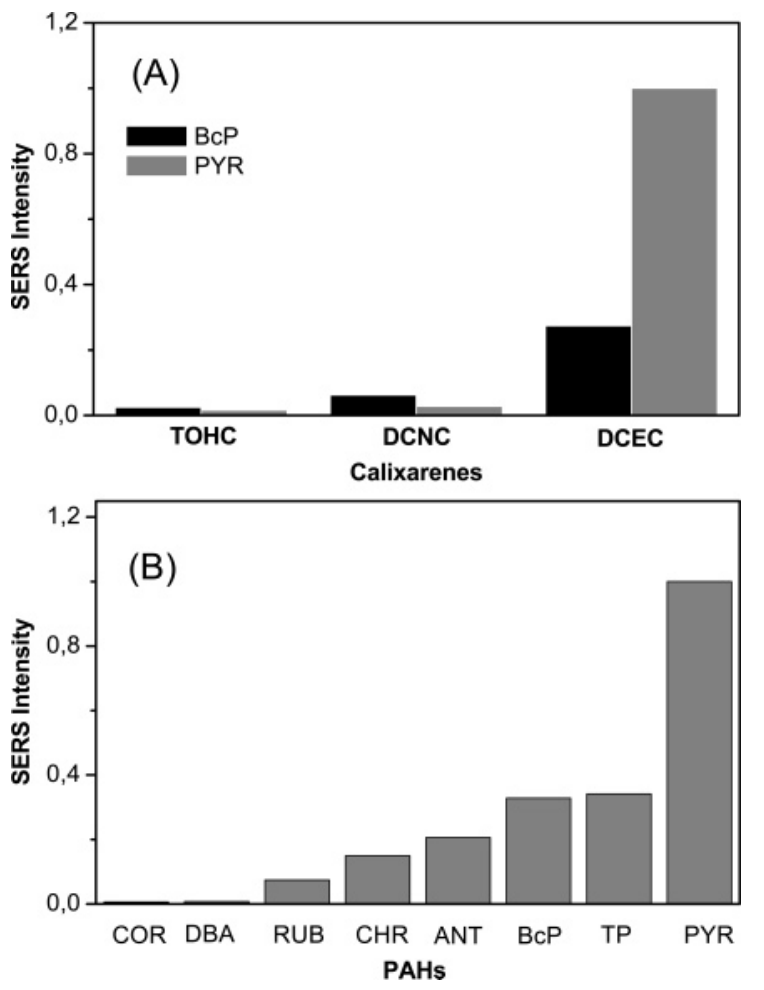

Figure 8. (A) Normalized areas (with respect to the $1405-\mathrm{cm}^{-1}$ band of PYR in the complex with DCEC) of the most intense SERS bands of PYR and TP $\left(10^{-4} \mathrm{M}\right)$ on the different assayed calixarenes. (B) Normalized areas (with respect to the $1405-\mathrm{cm}^{-1}$ band of PYR in the complex with DCEC) of the most intense SERS bands of the studied PAHs $\left(10^{-4} \mathrm{M}\right)$ on DCEC $\left(10^{-4} \mathrm{M}\right)$. All of the intensities were registered by exciting at $1064 \mathrm{~nm}$.

TOHC and DCNC. In contrast, BcP also gives rise to a high SERS intensity when interacting with DCEC, although lower than that reached by PYR, whereas it displays a significant SERS intensity when interacting with the other calixarenes. This result indicates the existence of a clear selectivity in the molecular recognition of PAHs by calixarenes thanks to the formation of a host/guest complex in which the chemical structure and the size of the PAHs play an important role. PYR seems to interact with a high specificity with DCEC. Because the differences between the assayed calixarenes are based on a different chemical functionalization on the lower rim, through which the calixarene interacts with the surface, the specificity could be due to a different adsorption affinity of the host/guest complex.

In Figure 8b, the normalized areas of the most intense SERS bands of the different PAHs studied here (the in-plane ringstretching band falling in the $1420-1320-\mathrm{cm}^{-1}$ region) were quantified in their complexes with DCEC. As can be seen, all of the four-ringed PAHs assayed (PYR, TP, and $\mathrm{BcP}$ ) are selectively more enhanced. In particular, the PYR, followed by the TP, are the PAH molecules with the highest specificity to DCEC because they lead to the maximum SERS intensification. In contrast, the larger PAHs (COR, RUB, and DBA) displayed the lowest SERS intensities. This size specificity is probably determined by the cavity of the calixarene and the tert-butyl groups placed in the upper rim. We note that CHR, another phenantrene derivative, leads to a much lower SERS intensity than $\mathrm{BcP}$. This indicates that the binding properties of each PAH must change depending on its chemical structure, revealing the existence of a size and shape selectivity of DCEC to interact with PAH molecules. 
Calixarenes bearing a dicarboethoxy $\left(-\mathrm{CH}_{2}-\mathrm{COO}-\mathrm{CH}_{2}-\right.$ $\mathrm{CH}_{3}$ ) substituent in the lower rim are then good candidates in the design of optical sensors of PAHs based on vibrational spectroscopy. The presence of these groups in an alternate configuration in the lower rim together with the tert-butyl groups in the upper rim ensure a strong interaction with both the ligand and the metal surface. This chemical structure is important for two possible reasons: (a) the existence of internal $\mathrm{H}$ bonds between the $-\mathrm{OH}$ of nonsubstituted phenolic groups placed in 2,4 positions, which could stabilize the cone conformation in DCEC necessary for the interaction with the PAH and which facilitates the interaction with the metal surface, as has been also found for other calixarenes ${ }^{41,42}$ and (b) the establishment of a strong interaction of DCEC with the metal surface or a better interaction with $\mathrm{Ag}^{+}$ions or clusters or adatoms, which seem to exist on the metal surface. ${ }^{43}$ The latter interaction is particularly strong in the case of ester-functionalized calixarenes, as was found by other authors, ${ }^{44,45}$ and could not take place in the case of DCNC calixarene. In previous work, ${ }^{46}$ we have also demonstrated that the existence of ester groups in polyterephthalate polymers could induce a strong interaction of the adsorbate molecule with the surface through the ester and ring moieties.

\section{Conclusions}

We have found that calixarenes can be successfully used in the detection of PAHs at trace concentrations by means of SERS spectroscopy on different substrates: Ag metal colloidal suspensions and nanoparticle immobilized films. The calixarene host molecule adsorbed onto the metal surface captures the PAH molecule close enough to the surface for the SERS detection. DCEC is the most appropriate host, among the calix[4]arene molecules assayed in this work, with regard to both the sensitivity and selectivity of the PAH detection. SERS spectral features of DCEC demonstrate that this molecule is adsorbed on the metal forming a self-assembly film on the colloidal nanoparticles and that the interaction with the $\mathrm{PAH}$ induces a significant change in the DCEC structure. From the analysis of the SERS spectra, useful information can be obtained about the importance of each molecular group in the calixarene affinity for PAHs and metal surfaces. In particular, the results presented here clearly demonstrate that the ester functionalization of the lower rim in calixarenes and the tert-butyl groups in the upper rim play an important role in the adsorption on the metal surface and the size-sensitive molecular recognition of PAHs by calixarenes. Thus, calix[4]arenes functionalized in this way can be employed in the design of highly sensitive and selective sensors of PAHs. DCEC was found to have a higher affinity for PYR and BcP. However, we believe that by modifying the calixarenes structure (i.e., a different number of benzene rings and a different substitution pattern in both the upper and the lower rim) one could modify their selectivity and viability in the application of these interesting molecules in optical detectors.

Acknowledgment. We acknowledge project Fondecyt 1040640 from Conicyt (Chile), the Convenio Conicyt/CSIC 2003/2004, project BFM/2001-2265 from Dirección General de Investigación, Ministerio de Ciencia y Tecnología (Spain), and project 07G/0042/2003 from Comunidad Autónoma de Madrid for financial support. P.L. acknowledges project AT 4040084 from Conicyt.

\section{References and Notes}

(1) Houk, K. N.; Leach, G. L.; Kim, S. P.; Zhang, X. Angew. Chem., Int. Ed. 2003, 42, 4872 .
(2) Gutsche C. D. In Calixarenes; Stoddart, J. F., Ed.; Monographs in Supramolecular Chemistry; Royal Society of Chemistry: Cambridge, England, 1992.

(3) Gutsche C. D.; Levine, J. A.; No, K. H.; Bauer, L. Tetrahedron 1983, 39, 409.

(4) Park, K. S.; Jung, S. O.; Lee, S. S.; Kim, J. S. Bull. Korean Chem. Soc. 1996, 21, 909.

(5) Marenco, C.; Stirling, C. J.; Yarwood, J. J. Raman Spectrosc. 2001, 32,183 .

(6) Kim, J. H.; Kim, Y. G.; Lee, K. H.; Kang, S. W.; Koh, K. N. Synth. Mater. 2001, 117, 145.

(7) Zhang, M.; Anderson, M. R. Langmuir 1994, 10, 2807.

(8) Throughton, E. B.; Bain, C. D.; Whitesides G. M. Langmuir 1988, 4,365 .

(9) http://www.inchem.org/documents/ehc/ehc/ehc202.htm.

(10) Van Gijssel, H. E.; Schild, L. J.; Watt, D. L.; Roth, M. J.; Wang, G. Q.; Dawsey, S. M.; Albert, P. S.; Qiao, Y. L.; Taylor, P. R.; Dong, Z. W.; Poirier, M. C. Mutat. Res. 2004, 547, 55.

(11) Falco, G.; Domingo, J. L.; Llobet, J. M.; Teixido, A.; Casas, C.; Muller, L. J Food Prot. 2003, 66, 2325.

(12) Guo, H.; Lee, S. C.; Chan, L. Y.; Li, W. M. Environ. Res. 2004, $94,57$.

(13) Li, D.; Jiao, L. Int. J. Gastrointest. Cancer 2003, 33, 3.

(14) Vo-Dinh, T.; Fetzer, J.; Campiglia, A. D. Talanta 1998, 47, 943.

(15) Stewart, S. D.; Fredericks, P. M. J. Raman Spectrosc. 1995, 26 , 629.

(16) Vasilyuk, G.; Maskevitch, S.; Podtynchenko, S. J. Mol. Struct. 2001 $565-566,389$.

(17) Carrasco, E. A.; Campos-Vallette, M.; Leyton P.; Diaz, G.; Clavijo, R. E.; García-Ramos, J. V.; Inostroza, N.; Domingo, C.; Sanchez-Cortes, S.; Koch, R. J. Phys. Chem. A 2003, 107, 9611.

(18) Moskovits, M. Rev. Mod. Phys. 1985, 57, 783

(19) Arenas, J. F.; Fernandez, D. J.; Soto, J.; Lopez-Tocon, I.; Otero, J. C. J. Phys. Chem. B 2003, 107, 13143.

(20) Wheling, B.; Hill, W.; Klockow, D. Int. J. Environ. Anal. Chem. 1999, 73, 223.

(21) Faull, J. D.; Gupta, V. K. Thin Solid Films 2003, 440, 129.

(22) Faull, J. D.; Gupta, V. K. Langmuir 2002, 18, 6584.

(23) Iwamoto, K.; Shinkai, S. J. Org. Chem. 1992, 57, 7066.

(24) Leopold, N.; Lendl, B. J. Phys. Chem. B 2003, 107, 2723.

(25) Sanchez-Cortes, S.; García-Ramos, J. V. J. Raman Spectrosc. 1998 29,365 .

(26) Sanchez-Cortes, S.; García-Ramos, J. V.; Morcillo, G.; Tinti, A. J. Colloid Interface Sci. 1995, 175, 358.

(27) Kondyurin, A.; Rautenberg, C.; Steiner, G.; Habicher, W. D.; Salzer, R. J. Mol. Struct. 2001, 563-564, 503.

(28) Katsyuba, S. A.; Grunenberg, J.; Schmutzler, R. J. Mol. Struct 2001, 559, 315.

(29) Katsyuba, S. A.; Schmutzler, R.; Hohm, U.; Kunze, C. J. Mol. Struct. 2002, 610, 113.

(30) Nakamoto, K. Infrared and Raman Spectra of Inorganic and Coordination Compounds. Part B: Applications in Coordination, Organometallic, and Bioinorganic Chemistry; John Wiley \& Sons: New York, 1997; p 59

(31) Mohammed-Ziegler, I. J. Spectrochim. Acta, Part A 2003, 59, 19.

(32) Hudgins, D. M.; Sandford, S. A. J. Phys. Chem. A 1998, 102, 329

(33) Shinohara, H.; Yamakita, Y.; Ohno, K. J. Mol. Struct. 1998, 442, 221.

(34) Carrasco, E. A.; Clavijo, R. E.; Campos-Vallette, M.; Leyton P.; Diaz, G.; Koch, R. Vib. Spectrosc. In press, 2004.

(35) Creighton, J. A. The Selection Rules for Surface-Enhanced Raman Spectroscopy in Spectroscopy of Surfaces; John Wiley \& Sons: Chichester, England, 1988; p 76.

(36) Kook, S. K. Bull. Korean Chem. Soc. 2002, 23, 111.

(37) Bhattacharya, S.; Nayak, S. K.; Chattopadhyay, S.; Banerjee, M.; Mukherjee, A. K. J. Phys. Chem. A 2003, 107, 11830.

(38) Kawaguchi, M.; Ikeda, A.; Shinkai, S.; Neda, I. J. Inclusion Phenom. Macrocyclic Chem. 2000, 37, 253.

(39) Asher, S. A.; Johnson, C. R. Science 1984, 225, 311.

(40) Dijkstra, R. J.; Martha, C. T.; Ariese, F.; Brinkman, U. A. Th.; Gooijer, C. Anal. Chem. 2001, 73, 4977.

(41) Rudkevich, D. M. Chem.-Eur. J. 2000, 6, 2679.

(42) Cho, Y. L.; Rudkevich, D. M.; Shinanyuk, A.; Rissanen, K.; Rebek, J., Jr. Chem.-Eur. J. 2000, 6, 3788

(43) Otto, A.; Mrozek, I.; Grabhorn, H.; Akemann, W. J. Phys.: Condens. Matter 1992, 4, 1143.

(44) Marcos, P. M.; Ascenso, J. R.; Segurado, M. A. P.; Pereira, J. L. C. J. Inclusion Phenom. Macrocyclic Chem. 2002, 42, 281.

(45) Ohto, K.; Murakami, E.; Shinohara, T.; Shiratsuchi, K.; Inoue, K.; Iwasaki, M. Anal. Chim. Acta 1997, 341, 275.

(46) Pérez-Mendez, M.; Marsal-Berenguer, R.; Sanchez-Cortes, S. Appl. Spectrosc. 2004, 58(5), 562. 